\title{
Advanced Topics in Modeling, Bifurcation Analysis, and Control Theory of Complex Systems
}

\author{
Olfa Boubaker $\left(\mathbb{D},{ }^{1}\right.$ Sajad Jafari $\left(\mathbb{D},{ }^{2}\right.$ Christos Volos $\mathbb{D},{ }^{3}$ Zeraoulia Elhadj, ${ }^{4}$ \\ Viet-Thanh Pham $(1),{ }^{5}$ and Jinde Cao $\mathbb{1}^{6}$ \\ ${ }^{1}$ National Institute of Applied Sciences and Technology, Tunis, Tunisia \\ ${ }^{2}$ Amirkabir University of Technology, Tehran, Iran \\ ${ }^{3}$ University of Thessaloniki, Thessaloniki, Greece \\ ${ }^{4}$ University of Tébessa, Tébessa, Algeria \\ ${ }^{5}$ Hanoi University of Science and Technology, Hanoi, Vietnam \\ ${ }^{6}$ Southeast University, Nanjing, China \\ Correspondence should be addressed to Olfa Boubaker; olfa.boubaker@insat.rnu.tn
}

Received 22 November 2017; Accepted 23 November 2017; Published 26 February 2018

Copyright (C) 2018 Olfa Boubaker et al. This is an open access article distributed under the Creative Commons Attribution License, which permits unrestricted use, distribution, and reproduction in any medium, provided the original work is properly cited.

In the recent years, several complex systems have been investigated in nonlinear theory and have had a huge impact on the scientist researches. Due to the complexity of their design, analysis, and control, innovative insights had emerged in this expanding area.

The purpose of this special issue is to draw attention of the scientific community to recent advances in nonlinear systems theory and their possible applications. The special issue includes several high-quality papers written by leading and emerging specialists in the field. It proposes an array of novel complex models belonging to the class of chaotic/hyperchaotic systems, fractional-order nonlinear systems, game systems, and smart grid and multiagent systems. Stability analyses and bifurcation investigations are also exposed for several models. Advanced control techniques and novel synchronization approaches are moreover suggested. We can quote, in that sense, applications not only related to the technology such as electronics and circuit design, multimedia, networks, and robotics but also related to biomedical and market domains.

More than 37 submissions from 21 countries (Algeria, Cameroon, China, Egypt, Ethiopia, Greece, Honduras, Hong Kong, Iran, Iraq, Lithuania, Mexico, Pakistan, Poland, Saudi Arabia, Spain, Qatar, Thailand, Tunisia, Turkey, USA, and Vietnam) have been received. Among the articles collected, a large number of high-quality papers existed, which led to 18 published articles with an acceptance rate of $45 \%$ (higher than that of the journal). This contribution reflects the strong interest in the considered subject.

Among the published papers in the special issue, three research papers propose novel hyperchaotic/chaotic systems supported by engineering applications and three other papers solve innovative chaos synchronization problems. Three papers propose bifurcation investigations of complex models related to biomedical applications and another three papers present bifurcation analysis and control of game models. Finally, there are two papers focusing on control of multiagent systems, two papers on control of robotic systems in presence of uncertainties, and two papers on control of coupled oscillators by external forcing. A very short description of the addressed topics, in the order of themes cited below, is presented as follows.

In "Dynamic Analysis and Circuit Design of a Novel Hyperchaotic System with Fractional-Order Terms," by A. Lassoued and O. Boubaker, a novel hyperchaotic system with fractional-order polynomials is designed. Its highly complex dynamics are investigated in terms of equilibrium points, Lyapunov spectrum, and attractor forms and a related electronic circuit is designed. 
In "Multimedia Security Application of a Ten-Term Chaotic System without Equilibrium," by X. Wang et al., a new chaotic system without equilibrium is proposed. The system is realized on an electronic card and applied in multimedia security, such as image encryption and sound steganography.

In "A New Nonlinear Chaotic Complex Model and Its Complex Antilag Synchronization,” by E. E. Mahmoud and F. S. Abood, a new chaotic Lü model with complex factors is covered and a novel antilag synchronization approach is also proposed.

In "Chaos Control in Fractional Order Smart Grid with Adaptive Sliding Mode Control and Genetically Optimized PID Control and Its FPGA Implementation," by A. Karthikeyan and K. Rajagopal, a specific smart grid system and its nonlinear properties are investigated. The Lyapunov exponents prove the existence of chaos and bifurcation. A related fractional order model is then derived and its bifurcation analysis is also investigated. Two different control methods are finally proposed to suppress the chaotic oscillations.

In "Centralized and Decentralized Data-Sampling Principles for Outer-Synchronization of Fractional-Order Neural Networks," by J.-E. Zhang, new sufficient criteria for an outersynchronization of a fractional-order neural networks are derived using centralized and decentralized data-sampling principles.

In "Master-Slave Synchronization of 4D Hyperchaotic Rabinovich Systems," by K. Ding et al., some original masterslave synchronization criteria are derived for 4D hyperchaotic Rabinovich systems and then extended for 3D chaotic Rabinovich systems.

In "Mixed Stimulus-Induced Mode Selection in Neural Activity Driven by High and Low Frequency Current under Electromagnetic Radiation," by L. Lu et al., a new Hindmarsh-Rose neuron model with different types of electrical stimulus impended with a high-low frequency current is proposed and deeply analyzed with regard to the transmission of several complex signals.

In "Computer Simulation of Noise Effects of the Neighborhood of Stimulus Threshold for a Mathematical Model of Homeostatic Regulation of Sleep-Wake Cycles," by W. Jin et al., noise effects on sleep-wake cycles' neuronal mathematical model determined by the hypocretin/orexin and the local glutamate interneurons spatiotemporal behaviors are analyzed. The conductance and the activation variable of the modulation function are also investigated based on a circadian input skewed in sine function.

In "Autaptic Modulation of Electrical Activity in a Network of Neuron-Coupled Astrocyte," by S. Guo et al., a simple network is developed for the Hodgkin-Huxley neuron coupled by astrocyte and the autapse and different modes of electrical activities and oscillating behaviors are deeply analyzed.

In "Study on Triopoly Dynamic Game Model Based on Different Demand Forecast Methods in the Market," by J. Ma et al., a new model of Triopoly game considering inaccurate demand in the market is proposed. Bifurcation dynamics, route to chaos, and basins of attraction are investigated. Finally, a feedback control method is suggested.
In "Complexity Dynamic Character Analysis of Retailers Based on the Share of Stochastic Demand and Service," by J. $\mathrm{Ma}$ et al., a modified price and demand game model based on the stochastic demand and the retailer's service level is proposed. The bifurcation phenomena as well the chaotic characteristics are shown. Finally, the chaos control of the complex model is achieved.

In "Nonlinear Complex Dynamics of Carbon Emission Reduction Cournot Game with Bounded Rationality," by L. Zhao, a novel Cournot duopoly game model of carbon emission reduction based on the hypothesis of participant's bounded rationality is proposed. The dynamic adjustment mechanism of emission reduction for enterprises is analyzed. It is shown that the system can demonstrate chaotic phenomenon. Finally, a delay feedback control is designed to control the system's complex dynamics.

In "A Novel Clustering Method Based on QuasiConsensus Motions of Dynamical Multiagent Systems," by N. Cai et al., a novel approach for clustering based on quasi-consensus of dynamical high-order multiagent systems is suggested and a necessary and sufficient condition for checking the achievement of group consensus is given.

In "Leader-Follower Fixed-Time Group Consensus Control of Multiagent Systems under Directed Topology," by Y. Shang and Y. Ye, the fixed-time group consensus problem is investigated for a leader-follower network of integrators with directed topology. A nonlinear distributed control protocol, based on local information, is proposed such that the follower agents in each subgroup are able to track their corresponding leaders in a prescribed convergence time regardless of the initial conditions.

In "Design of Robust Supertwisting Algorithm Based Second-Order Sliding Mode Controller for Nonlinear Systems with Both Matched and Unmatched Uncertainty," by M. Jouini et al., a modified design of the super-twisting controller for nonlinear systems with matched and unmatched uncertainties is proposed. The main contribution resides in adding two terms to the classical design which leads to accelerating the convergence and simultaneously limiting the overshoot and shortening the settling time of the system response.

In "Tracking Control for Mobile Robots Considering the Dynamics of All Their Subsystems: Experimental Implementation," by J. R. Garcia-Sanchez et al., the trajectory tracking task in a wheeled mobile robot is solved by proposing a three-level hierarchical controller that considers the mathematical model of the mechanical structure, actuators, and power stage. Experimental results show the effectiveness and robustness of the proposed control scheme.

In "Multistability Analysis and Function Projective Synchronization in Relay Coupled Oscillators," by A. T. Azar et al., regions of stability are discovered in a general class of Genesio-Tesi chaotic oscillators for which attractors and limit cycles are shown and isospike diagrams are also exposed. Finally, finite-time projective synchronization of relay coupled oscillators is displayed.

In "Stabilization of a Network of the FitzHugh-Nagumo Oscillators by Means of a Single Capacitor Based RC Filter Feedback Technique," by E. Adomaitienè et al., an original control approach for unstable FitzHugh-Nagumo oscillators 
is proposed based on analytical, numerical, and also experimental investigations. It is shown that a single capacitor is sufficient to achieve this task if the oscillators are coupled strongly enough.

\section{Acknowledgments}

The editors would like to express their thanks to all authors of the special issue for their valuable contributions and to all reviewers for their useful efforts to provide valuable reviews. We expect this special issue to offer a timely view of advanced topics in nonlinear systems, which will grant stimulation for further novel academic researches and innovative applications.

Olfa Boubaker Sajad Jafari Christos Volos Zeraoulia Elhadj Viet-Thanh Pham Jinde Cao 


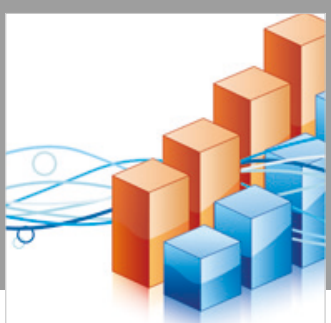

Advances in

Operations Research

\section{-n-m}
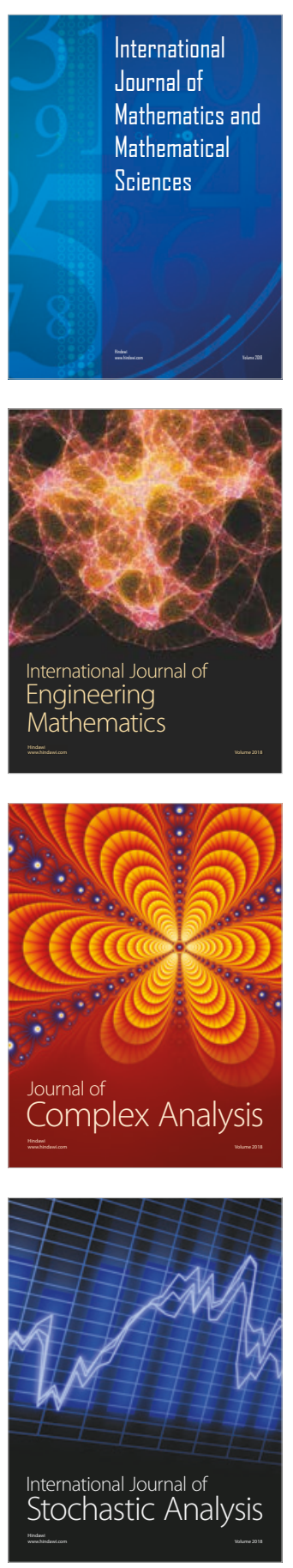
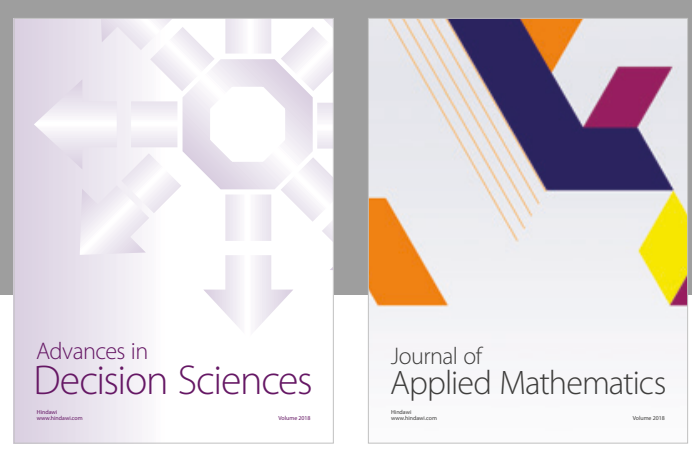

Journal of

Applied Mathematics
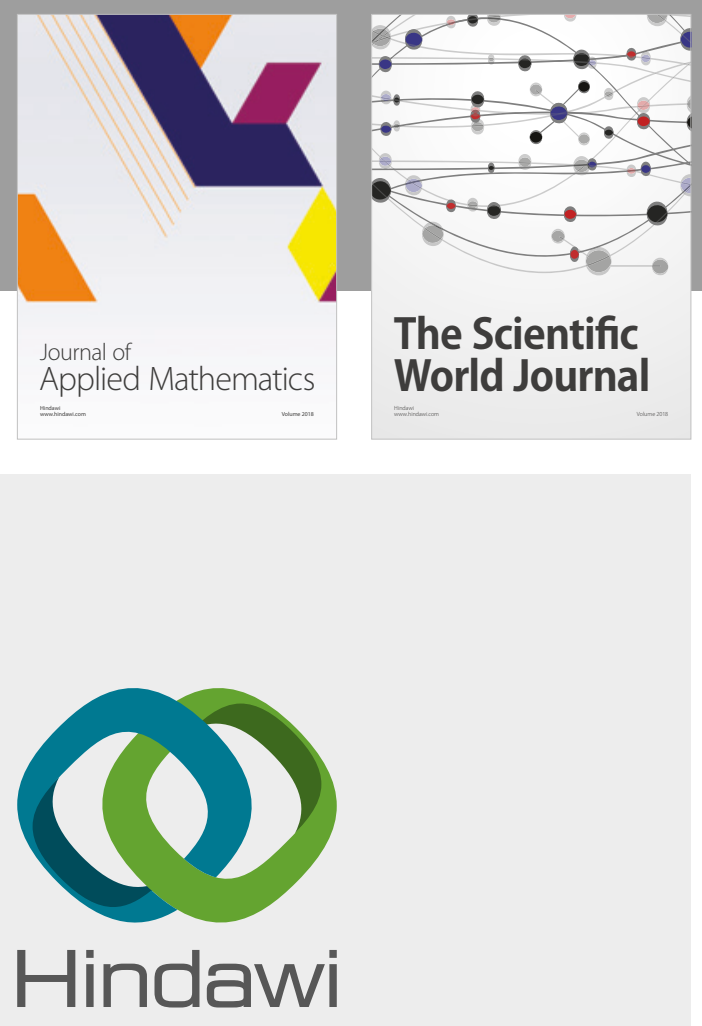

Submit your manuscripts at

www.hindawi.com

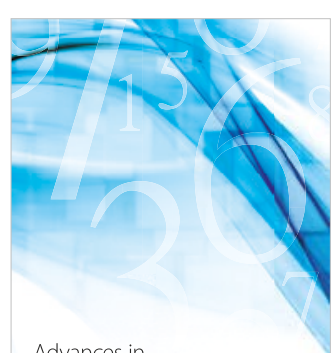

Advances in
Numerical Analysis
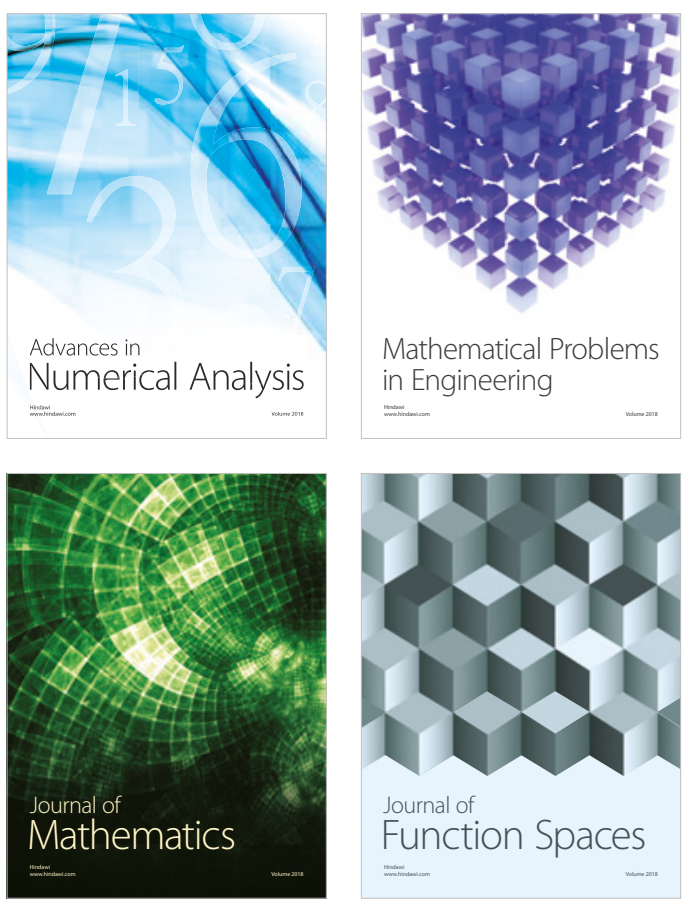

Mathematical Problems in Engineering

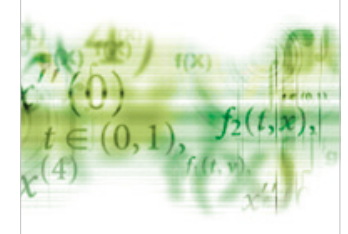

International Journal of

Differential Equations

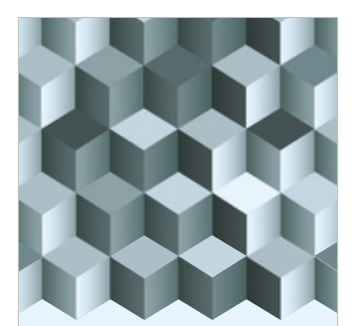

Journal of

Function Spaces
The Scientific

World Journal

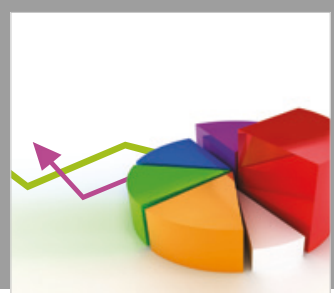

Journal of

Probability and Statistics
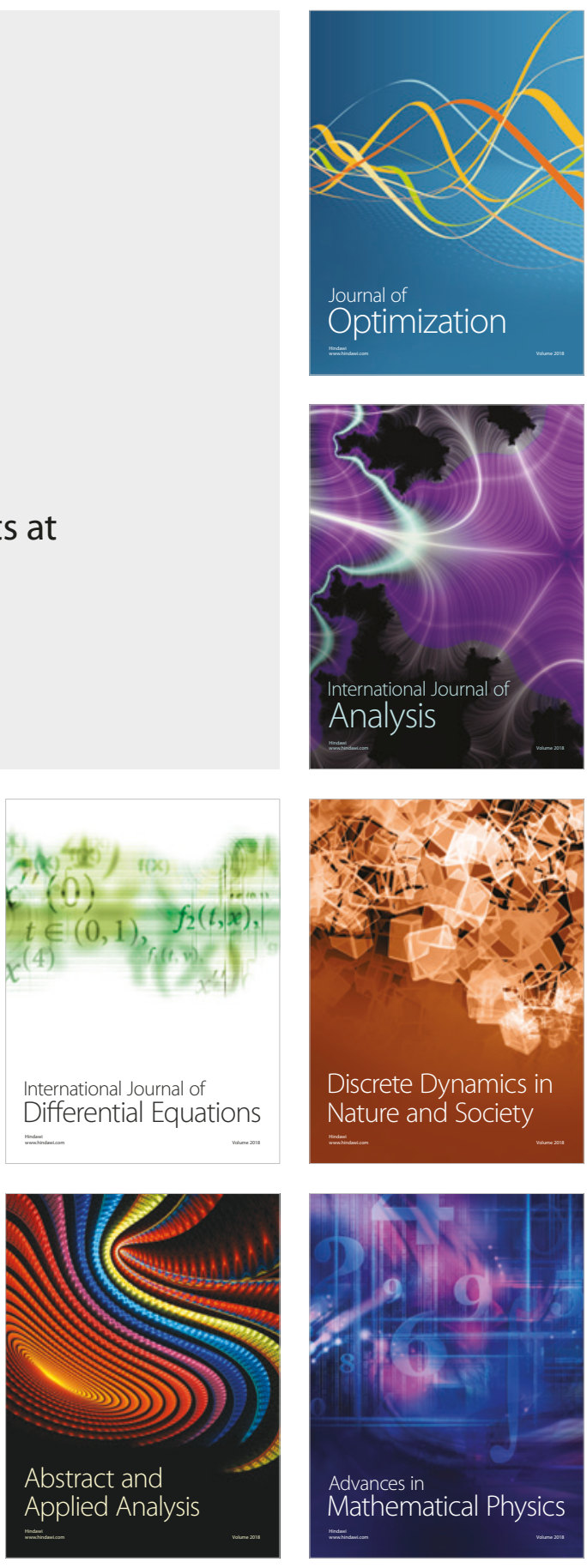